\title{
New hydroelastic solitary waves in deep water and their dynamics
}

\author{
T. Gao ${ }^{1}$, Z. Wang ${ }^{2} \uparrow$ and J. -M. Vanden-Broeck ${ }^{1}$ \\ ${ }^{1}$ Department of Mathematics, University College London, London, WC1E 6BT, UK \\ ${ }^{2}$ Department of Mathematical Sciences, University of Bath, Bath, BA2 7AY, UK
}

(Received )

A numerical study of fully nonlinear waves propagating through a two-dimensional deep fluid covered by a floating flexible plate is presented. The nonlinear model proposed by Toland (2008) is used to formulate the pressure exerted by the thin elastic sheet. The symmetric solitary waves previously found by Guyenne \& Părău (2012) and Wang et al. (2013) are quickly reviewed. A new class of hydroelastic solitary waves which are nonsymmetric in the direction of wave propagation is then computed. These asymmetric solitary waves have a multi-packet structure and appear via spontaneous symmetry-breaking bifurcations. We study in detail the stability properties of both symmetric and asymmetric solitary waves subject to longitudinal perturbations. Some moderate-amplitude symmetric solitary waves are found to be stable. A series of numerical experiments are performed to show the non-elastic behaviour of two interacting stable solitary waves. The large response generated by a localised steady pressure distribution moving at a speed slightly below the minimum of the phase speed (called transcritical regime in the literature) is also examined. The direct numerical simulation of the fully nonlinear equations with a single load reveals that in this range the generated waves are of finite amplitude. This includes a perturbed depression solitary wave, which is qualitatively similar to the large response observed in experiments. The excitations of stable elevation solitary waves are achieved by applying multiple loads moving with a speed in the transcritical regime.

Key words: elastic waves, solitary waves, surface gravity waves

\section{Introduction}

Hydroelasticity, a name adapted from aeroelasticity, is concerned with the motion and distortion of deformable bodies responding to hydrodynamic excitations, and the associated reactions on the motion of the environmental fluid. Hydroelastic waves enjoy wide usages in marine structures and sea transport. Modern applications of hydroelastic waves abound: very large floating structures usable as fully functional airport runways (Megafloat project in Japan); large fast merchant ships and container vessels which are relatively more flexible (Wang (2000)); flexible risers to transport hydrocarbon (mainly refers to oil) from the seabed to shore or offshore facilities (Jain (1994)); safe use of lake and ocean ice for roadways and landing strips (Wilson (1958); Takizawa (1985); Squire et al. (1988)). Due to these physical and industrial significances, in-depth knowledge of the characteristics of hydroelastic waves is therefore important.

The present work considers the irrotational motion of a two-dimensional inviscid and

$\dagger$ Email address for correspondence: z.wang5@bath.ac.uk 
incompressible fluid of infinite depth, with the top surface being in contact with a frictionless thin elastic sheet. Since there are two restoring forces across the free surface (the gravity and the flexural rigidity due to the elastic bending) the hydroelastic waves propagating through the elastic cover are also called flexural-gravity waves. This problem has been proposed as an ideal model for the dynamics of a large body of waves covered by a floating ice sheet in polar regions, and has been studied extensively mostly in the linear setting since the pioneering work by Greenhill (1886) (the reader is referred to the monograph by Squire et al. (1996) which describes in detail the research based on the linear theory prior to 1996).

The linear theory is a good approximation when the deformations of the elastic cover are relatively small, but it becomes unreliable as the amplitude of the waves grows (the reader is referred to the reports of intense-in-ice events by Marko (2003) which highlight the limitations of the linear theory). In particular, the linear theory fails for the problem of a concentrated line load moving steadily over an ice sheet floating on a fluid when the velocity of the load is at the minimum of the phase speed (denoted by $c_{\text {min }}$ ). This breakdown of the linear theory is due to an accumulation of the energy which makes the displacement of the free surface grow without limit. In order to resolve the problem, Părău \& Dias (2002) developed a nonlinear theory valid when the speed of the load is close to the critical value $c_{\text {min }}$. They conducted a normal form analysis which leads to a forced nonlinear Schrödinger equation (NLS) with the mean depth of the fluid being a parameter. Their results show that for water of sufficiently large depth, bounded responses in the form of hydroelastic solitary waves can exist for speeds up to $c_{\min }$, while there is a range of forcing speed below $c_{\text {min }}$ for which there are no steady solutions when the fluid is relatively shallow. Unsteady simulations using truncated models and high-order spectral methods were carried out by Bonnefoy et al. (2009), confirming the predictions of Părău \& Dias (2002) in the appropriate regime. Milewski et al. (2011) revisited the same problem and found hydroelastic solitary waves in deep water in the absence of moving loads, even though the weakly nonlinear analysis shows that the associated cubic NLS is of defocussing type at the minimum of the phase speed. However these solitary waves are unusual, since they can only exist with finite amplitudes. In other words, they do not bifurcate from infinitesimal periodic waves like the gravity-capillary solitary waves in deep water (see, e.g., Vanden-Broeck \& Dias (1992); Wang et al. (2014)), nor from infinitesimal long waves like the Korteweg-de Vries (KdV) equation. Furthermore, the unsteady simulations presented in the same paper reveal that these solitary waves may arise naturally from the moving load problem subject to a moderateamplitude near-critical forcing. This fact indicates that the existence and the stability of free solitary waves are crucial for the understanding of the forced problem.

All the results mentioned in the last paragraph are based on the Kirchhoff-Love model which uses $\partial_{x x} \kappa$ to model the pressure due to the bending of the elastic sheet, where $\kappa$ is the curvature of the surface and $x$ is the coordinate in the direction of wave propagation. The Kirchhoff-Love elastic model has also been used in the absence of moving loads, to study periodic hydroelastic waves (Forbes $(1986,1988)$ ), generalised flexural-gravity solitary waves (Vanden-Broeck \& Părău (2011)) and the unsteady interaction between a fluid-loaded elastic plate and a mean flow (Peake (2001)). Although the KirchhoffLove model is widely used in the literature it has some limitations. In particular it does not appear to have an elastic potential. More recently, Toland (2007, 2008) proposed a novel nonlinear elastic model using the Cosserat theory of hyperelastic shells satisfying Kirchhoff's hypotheses, which has a clear variational structure. From then on, the analytical and numerical investigations of this new model have been gradually carried out. Of note are the works of Toland (2008) who rigorously proved the existence of periodic 
hydroelastic waves, Guyenne \& Părău (2012) who discovered that both elevation and depression branches exist below the minimum of phase speed at finite amplitude in deep water, Wang et al. (2013) who extended the branch of elevation solitary waves to the highly nonlinear regime with the wave profiles featuring multi-packet structure and computed periodic waves with an overhanging structure, Gao \& Vanden-Broeck (2014) who investigated the generalised solitary waves extensively, and Page \& Părău (2014) who considered nonlinear hydroelastic hydraulic falls past a submerged bottom obstruction.

There are relatively fewer studies on unsteady hydroelastic waves based on Toland's model. It is worth mentioning that Guyenne \& Părău performed direct numerical simulations for unsteady hydroelastic solitary waves in deep water (Guyenne \& Părău (2012)) and shallow water (Guyenne \& Părău (2014)). However their numerics is based on reduced models with the truncated Dirichlet-Neumann operator which cannot be used to study highly nonlinear waves, such as overhanging waves. To our knowledge, there have been no computations of the dynamics of hydroelastic solitary waves using the full Euler equations and Toland's model.

In this paper, we use a time-dependent conformal map technique to study numerically the bifurcation, stability, collision and excitation of hydroelastic solitary waves. We restrict our attention to deep water and to two dimensions. Motivated by the recent work of Wang et al. (2014), we compute a new branch of solitary waves which are non-symmetric in the direction of wave propagation. These waves have a multi-packet structure. We study numerically the stability of all hydroelastic solitary waves found to date. Our results show that some symmetric solitary waves are robust subject to longitudinal perturbations, including not only the moderate-amplitude single-trough depression solitary waves, but also certain elevation waves resembling two big troughs placed sideby-side. We show that these stable two-trough coherent structures can be excited by multiple loads simultaneously moving with a speed slightly below $c_{\text {min }}$.

The rest of the paper is structured as follows. We state the mathematical formulation and introduce the time-dependent conformal map technique used to reduce the dimension of the problem in $\S 2$ and $\S 3$ respectively. We review the properties of symmetric solitary waves in $\S 4.1$, and compute asymmetric solitary waves in $\S 4.2$. The stability properties for both symmetric and asymmetric waves are studied numerically in $\S 5$. We then consider the interaction between two stable solitary waves, including both head-on and over-taking collisions. The numerical experiments on the excitation of solitary waves by one or multiple constant-velocity loads are performed in $\S 6.2$. Concluding remarks and possible future projects are presented in the last section.

\section{Formulation}

We consider a two-dimensional irrotational flow of an incompressible and inviscid fluid beneath a thin elastic sheet. The fluid is assumed to be of infinite depth. We introduce cartesian coordinates with the $y$-axis directed vertically upwards and with $y=0$ at the undisturbed level of the elastic sheet. The deformation of the elastic sheet is denoted by $y=\eta(x, t)$ where $t$ is the time. Since the flow is irrotational we can introduce a potential function $\phi$, such that the velocity field reads $\left(\phi_{x}, \phi_{y}\right)$. The problem reduces then to solving Laplace's equation

$$
\phi_{x x}+\phi_{y y}=0, \quad \text { for }-\infty<y<\eta(x, t) .
$$

The main approximations made here are that the elastic sheet is thin, and that its inertia and its stretching (or the existence of a pre-stressed state) are neglected. Therefore the only restoring forces are gravity and the flexural elasticity of the elastic sheet. In the 
present paper, we use the fully nonlinear model based on the special Cosserat theory of hyperelastic shells introduced by Toland (2008). Therefore the nonlinear boundary conditions at $y=\eta(x, t)$ are the kinematic condition

$$
\eta_{t}=\phi_{y}-\eta_{x} \phi_{x}
$$

and the dynamic condition

$$
\phi_{t}=-\frac{1}{2}\left[\phi_{x}^{2}+\phi_{y}^{2}\right]-g \eta-\frac{\mathcal{D}}{\rho} \mathcal{P}_{b}+\mathcal{P}_{e}(x, t),
$$

where $g$ is the acceleration due to gravity, $\rho$ is the density of the fluid, $\mathcal{P}_{e}$ is the external pressure distribution exerted on the elastic sheet and $\mathcal{D}$ is the coefficient of flexural rigidity defined as $\mathcal{D}=E h^{3} / 12\left(1-\nu^{2}\right)$. Here $E$ is the Young's modulus, $\nu$ the Poisson ratio and $h$ the thickness of the elastic sheet. $\mathcal{P}_{b}$ is the pressure distribution due to elastic bending. It follows from Toland (2008) (equation (1.13) in Toland's paper when the surface tension is neglected) that

$$
\mathcal{P}_{b}=\kappa_{s s}+\frac{1}{2} \kappa^{3},
$$

where $\kappa$ is the curvature of the free surface and $s$ is the arc-length parameter. If the wave profile is single-valued, the curvature can be expressed in the Cartesian coordinates as

$$
\kappa=\frac{\eta_{x x}}{\left(1+\eta_{x}^{2}\right)^{3 / 2}} .
$$

Finally, the far-field condition

$$
\phi_{y} \rightarrow 0, \quad \text { as } y \rightarrow-\infty
$$

completes the formulation. The system can be non-dimensionalized by choosing

$$
\left[\frac{\mathcal{D}}{\rho g}\right]^{1 / 4}, \quad\left[\frac{\mathcal{D}}{\rho g^{5}}\right]^{1 / 8}, \quad\left[\frac{g \mathcal{D}^{3}}{\rho^{3}}\right]^{1 / 8}
$$

as the units of length, time and potential respectively.The dynamic boundary condition can be rewritten as

$$
\phi_{t}=-\frac{1}{2}\left[\phi_{x}^{2}+\phi_{z}^{2}\right]-\eta-\left(\frac{1}{2} \kappa^{2}+\kappa_{s s}\right)+\mathcal{P}_{e}(x, t) .
$$

The equations (2.1) (2.2) (2.4) (2.6) and (2.8) form a Hamiltonian system with the action functional being the total energy of the fluid which is the sum of the kinetic energy and the potential energy:

$$
\mathcal{E}=\frac{1}{2}\left[\int_{\mathbb{R}} d x \int_{-\infty}^{\eta}\left(\phi_{x}^{2}+\phi_{y}^{2}\right) d y+\int_{\mathbb{R}} \eta^{2} d x+\int \kappa^{2} d s\right] .
$$

Here $\mathbb{R}$ is the real line. We denote the velocity potential on the free surface by $\varphi(x, t) \triangleq$ $\phi(x, \eta(x, t), t)$. Working in the canonical variables $\varphi$ and $\eta$, the kinematic and dynamic boundary conditions can be recast as:

$$
\eta_{t}=\frac{\delta \mathcal{E}}{\delta \varphi}, \quad \varphi_{t}=-\frac{\delta \mathcal{E}}{\delta \eta} .
$$

It is noted that the effects due to inertia and the thickness of the elastic sheet can also be incorporated into the pressure equation (2.8) (see Squire et al. (1996) for the expression of the inertia and Forbes (1986) for how to model the system with the thickness of 
the plate). However the unsteady simulations become much more complicated when the inertia is taken into account. For simplicity we neglect these two effects, and only consider the pressure jump exerted by the elastic sheet owing to flexing. Even with these approximations, the hydroelastic wave problem has been modelled with a variety of methods. The reader is referred to Milewski \& Wang (2013) for a discussion.

\section{Numerical Method}

The main idea for solving two-dimensional full Euler equations is based on a timedependent conformal mapping. This numerical method was pioneered by Dyachenko et al. (1996). We start the derivation with finding a transformation that maps the physical domain $-\infty<y<\eta(x, t)$ into the lower half plane with horizontal and vertical coordinates denoted by $\xi$ and $\zeta$ respectively. The conformal mapping from $(x, y)$ to $(\xi, \zeta)$ can be found by solving the following boundary-value problem:

$$
\begin{cases}y_{\xi \xi}+y_{\zeta \zeta}=0 & \text { for }-\infty<\zeta<0 \\ y=Y(\xi, t) & \text { at } \zeta=0 \\ y \sim \zeta & \text { as } \zeta \rightarrow-\infty\end{cases}
$$

where $Y(\xi, t)=\eta(x(\xi, 0, t), t)$. The harmonic conjugate $x(\xi, \eta, t)$ is defined through the Cauchy-Riemann relations for the complex function $x(\xi, \zeta, t)+i y(\xi, \zeta, t)$. In the transformed plane, the velocity potential $\phi$ and its harmonic conjugate $\psi(\xi, \zeta, t)$ also satisfy Laplace's equations. Defining $\Phi(\xi, t) \triangleq \phi(x(\xi, 0, t), y(\xi, 0, t), t), \Psi(\xi, t) \triangleq \psi(\xi, 0, t)$ and $X(\xi, t) \triangleq x(\xi, 0, t)$ we obtain after some elementary analysis

$$
X=\xi-\mathcal{H}[Y] \quad \text { and } \quad \Psi=\mathcal{H}[\Phi],
$$

where $\mathcal{H}$ is the operator of Hilbert transformation with the Fourier $\operatorname{symbol} i \operatorname{sgn}(k)$. It can also be defined in the physical space as

$$
\mathcal{H}[f](\xi)=\mathrm{PV} \int_{\mathbb{R}} \frac{f\left(\xi^{\prime}\right)}{\xi^{\prime}-\xi} d \xi^{\prime},
$$

where 'PV' indicates the Cauchy principal value of the integral. By using the chain rule, the kinematic boundary condition in the transformed plane takes the form

$$
X_{\xi} Y_{t}-Y_{\xi} X_{t}=-\Psi_{\xi}
$$

Following Dyachenoko et al. (1996), the evolution equation for $Y$ is

$$
Y_{t}=Y_{\xi} \mathcal{H}\left[\frac{\Psi_{\xi}}{J}\right]-X_{\xi} \frac{\Psi_{\xi}}{J}
$$

where $J=X_{\xi}^{2}+Y_{\xi}^{2}$ is the Jacobian of the conformal map. Finally we reformulate the dynamic boundary condition using the new variable $\xi$ as

$$
\Phi_{t}=\frac{\Psi_{\xi}^{2}-\Phi_{\xi}^{2}}{2 J}-Y-\frac{1}{2}\left[\frac{\kappa_{\xi \xi}}{J}+\left(\frac{\kappa_{\xi}}{J}\right)_{\xi}+\kappa^{3}\right]+\Phi_{\xi} \mathcal{H}\left[\frac{\Psi_{\xi}}{J}\right]+\mathcal{P}_{e},
$$

where the curvature is now written as

$$
\kappa=\frac{X_{\xi} Y_{\xi \xi}-X_{\xi \xi} Y_{\xi}}{J^{3 / 2}}
$$

In order to find the dispersion relation of the system, we linearize the surface Euler system (3.5)-(3.6) by taking $Y, \Phi_{\xi}, \Psi_{\xi}$ small, $\mathcal{P}_{e}=0$ and $X_{\xi} \sim 1, J \sim 1$. This yields 
$\Phi_{t t}=\mathcal{H}\left[\Psi_{\xi}\right]+\mathcal{H}\left[\Psi_{\xi \xi \xi \xi \xi}\right]$. Therefore the dispersion relation is

$$
\omega^{2}=k^{2} c^{2}=|k|\left(1+k^{4}\right) .
$$

Relation (3.8) implies that the phase velocity $c$ reaches its minimum value $c_{\min } \approx 1.3247$ when $k=(1 / 3)^{1 / 4}$. The phase velocity is then equal to the group velocity at this minimum.

We seek fully localised wave solutions travelling with speed $c$. In order to guarantee that there are no waves in the far field, we choose $c<c_{\text {min }}$. We assume that the functions $Y$ and $\Phi$ depend on $\xi-c t$. It then follows from (3.4) that

$$
\Psi_{\xi}=c Y_{\xi} \text {, and hence } \Phi_{\xi}=-c \mathcal{H}\left[Y_{\xi}\right] .
$$

From (3.5), one can conclude that

$$
\mathcal{H}\left[\frac{\Psi_{\xi}}{J}\right]=c\left(\frac{X_{\xi}}{J}-1\right) .
$$

Substituting (3.9) and (3.10) into the dynamic boundary condition (3.6) with $\mathcal{P}_{e}=0$ (since we seek free solitary waves) and noticing that $\Phi_{t}=-c \Phi_{\xi}$, we obtain after some algebra the following nonlinear equation

$$
\frac{c^{2}}{2}\left(\frac{1}{J}-1\right)+Y+\frac{1}{2}\left[\frac{\kappa_{\xi \xi}}{J}+\left(\frac{\kappa_{\xi}}{J}\right)_{\xi}+\kappa^{3}\right]=0 .
$$

This, together with $X_{\xi}=1-\mathcal{H}\left[Y_{\xi}\right]$ defines an integro-differential system. Finally the velocity potential can be recovered from $\Phi=-c \mathcal{H}[Y]$. It is worth mentioning that the curvature (2.5) is only valid for single-valued solutions, while the formulation (3.11) based on the new parameter $\xi$ is even true for multivalued wave profile, and the system (3.5)(3.6) allows us to compute the evolution of overhanging waves. In the new coordinates, the Hamiltonian (2.9) becomes

$$
\mathcal{E}=\frac{c^{2}}{2} \int_{\mathbb{R}} Y_{\xi} \mathcal{H}[Y] d \xi+\frac{1}{2} \int_{\mathbb{R}} Y^{2} X_{\xi} d \xi+\frac{1}{2} \int_{\mathbb{R}} \frac{\left(Y_{\xi \xi} X_{\xi}-X_{\xi \xi} Y_{\xi}\right)^{2}}{J^{5 / 2}} d \xi .
$$

where the terms on the right-hand side correspond to kinetic energy, gravitational potential energy and elastic potential energy respectively. We remark that conformal mapping is a conventional technique to handle free-surface water wave problems in two dimensions. Equation (3.11) and its modified versions were widely used in computing periodic and solitary waves in deep water. Of note are the works of Crapper (1957) who obtained analytical solutions for pure capillary in terms of elementary functions, Longuet-Higgins (1988) and Schwarts \& Vanden-Broeck (1979) who computed different branches of periodic capillary-gravity waves, and Guyenne \& Părău (2012) who considered flexuralgravity solitary waves based on Toland's nonlinear elastic model. All the aforementioned works show that multivalued steady profiles exist in capillary/capillary-gravity/flexuralgravity waves, with a limiting configuration pitching off a closed bubble.

Details of the numerical procedure for finding fully localised travelling waves in (3.11) are given as follows. The solitary-wave solutions are approximated by long periodic waves. Therefore the equation (3.11) can be solved numerically via truncating the Fourier series of $Y$, namely,

$$
Y(\xi)=\sum_{n=0}^{N} a_{n} \cos (n \pi \xi / L)+\sum_{n=1}^{N} \sin (n \pi \xi / L) .
$$

The Fourier coefficients $a_{n}$ and $b_{n}$ need to be found so that the dynamic boundary 
condition (3.11) is satisfied. This is done numerically. We first discretise the domain $[-L, L)$ into a uniform mesh. We evaluate $(3.11)$ on the grid points and then project it onto each element of the $\cos (n \pi \xi / L)$ and $\sin (n \pi \xi / L)$ basis for $n=0,1, \cdots, N$. All the derivatives and the Hilbert transform are calculated via Fourier multipliers making the program efficient and accurate, while the nonlinear terms are computed in real space. The resultant system of nonlinear algebraic equations is solved by Newton's method. The underlying period $2 L$ and the total wavenumber $N$ are both chosen to be sufficiently large so that the wave profiles hardly change as $L$ and $N$ are further increased. In most computations, we keep the mesh size $\Delta \xi=0.05$. We stop the iterations when the $l^{\infty}$-norm of the residual error is less than $10^{-10}$. It is noted that for symmetric solitary waves $b_{n}=0$ for all $n$, which reduces considerably the computing time. As a validation of the numerical method, we recomputed the solutions of Crapper (1957) for periodic capillary waves of infinite depth. We obtained an excellent agreement with Crapper's solutionsfull bifurcation diagram which relates the wave speed and the wave amplitude (this is tested by checking that the $l^{\infty}$-norm of the errors are all less than $10^{-14}$ ). In particular we were able to compute the overhanging profiles which occur for waves close to the limiting configuration.

\section{Travelling Waves of Permanent Form}

\subsection{Symmetric Waves}

In this section we describe the basic properties of symmetric solitary waves propagating at a constant velocity $c$ without change of form. These waves will be used in $\S 4.2$ as building blocks to construct new asymmetric waves. The computation of steady symmetric solitary waves can also serve as a validation of the numerical method since we just reproduced the results in Guyenne \& Părău (2012) and Wang et al. (2013).

Guyenne \& Părău (2012) first computed free solitary waves for the Toland model. They found that there is an elevation branch (a positive free-surface elevation $\eta(0)$ at the centre of the wave) and a depression branch (a negative free-surface elevation $\eta(0)$ at the centre of the wave) bifurcating from the phase speed minimum $c^{*} \approx 1.3247$. They noticed that these solitary waves exist only with non-zero amplitude as shown in figure 1(a). A similar result was found earlier for the Kirchhoff-Love model (see Milewski et al. (2011)). Later, Wang et al. (2013) calculated the complete solution branches for depression and elevation solitary waves. They found that the elevation branch exhibits multiple turning points, which indicates that different elevation solitary waves can travel at the same speed (typical profiles of the elevation solitary waves are shown in figure 8(b)). For the depression branch, both Guyenne \& Părău (2012) and Wang et al. (2013) found that as the speed $c$ decreases and approaches zero, the wave profiles become steeper, eventually with an overhanging structure (a typical example of overhanging depression solitary waves is shown in the right panel of figure 6).

The two branches in figure 1 (a) bifurcate from the phase speed minimum $c^{*} \approx 1.3247$ with nonzero amplitudes. This is to be contrasted with the problem of gravity-capillary waves where the branches bifurcate from the phase speed minimum with $\eta(0)=0$ (see for example Vanden-Broeck \& Dias (1992)). The reason for this difference is that the associated nonlinear Schrödinger equation (NLS) is of defocussing type for the present problem (see Milewski \& Wang (2013) for details).

The speed-energy bifurcation curves shown in figures $1(\mathrm{~b}, \mathrm{c})$ are closely related to the stability properties of solitary waves subject to longitudinal perturbations. Saffman (1985) considered the stability of periodic waves due to superharmonic perturbations 

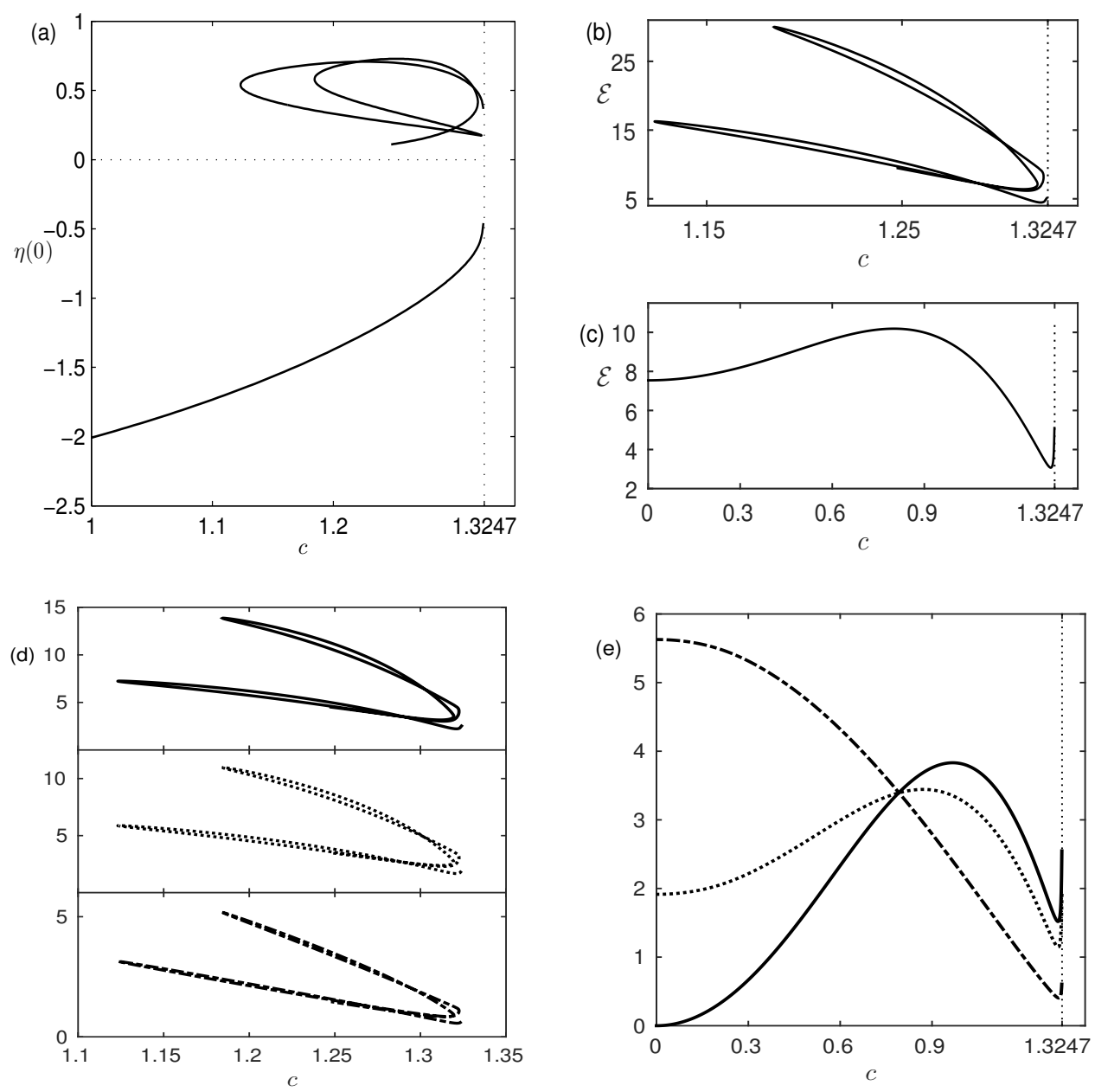

FIGURE 1. Speed-amplitude and speed-energy curves for symmetric elevation and depression solitary waves emerging from the bifurcation point $c^{*} \approx 1.3247$. (a) speed-amplitude curves of the elevation and depression branches. The value of $\eta$ at the middle point is considered as the amplitude. The depression branch is monotonic for $c \in\left[0, c^{*}\right)$ (only part of the curve is shown), while the elevation branch demonstrates a complex behaviour with multiple turning points. (b) speed-energy curve of the elevation branch showing a zig-zag behaviour; (c) speed-energy curve of the depression branch with two stationary points. The energy partition is shown in (d) for the elevation branch, and in (e) for the depression branch, where the total energy is partitioned into kinetic energy (solid line), gravitational potential energy (dotted line), and elastic potential energy (dash-dotted line).

(the period of the perturbation is less than that of the wave), and gave a necessary but not sufficient condition for the stability exchange. He pointed out that stability exchanges can only occur at critical points, either stationary points or turning points, of the speed-energy bifurcation curve, namely,

$$
\frac{\partial \mathcal{E}}{\partial c}=0 \text { or } \frac{\partial c}{\partial \mathcal{E}}=0
$$

Saffman's argument is based on the Hamiltonian formulation of water waves (see Zakharov (1968)) and is developed for pure gravity waves. However, the result can be 


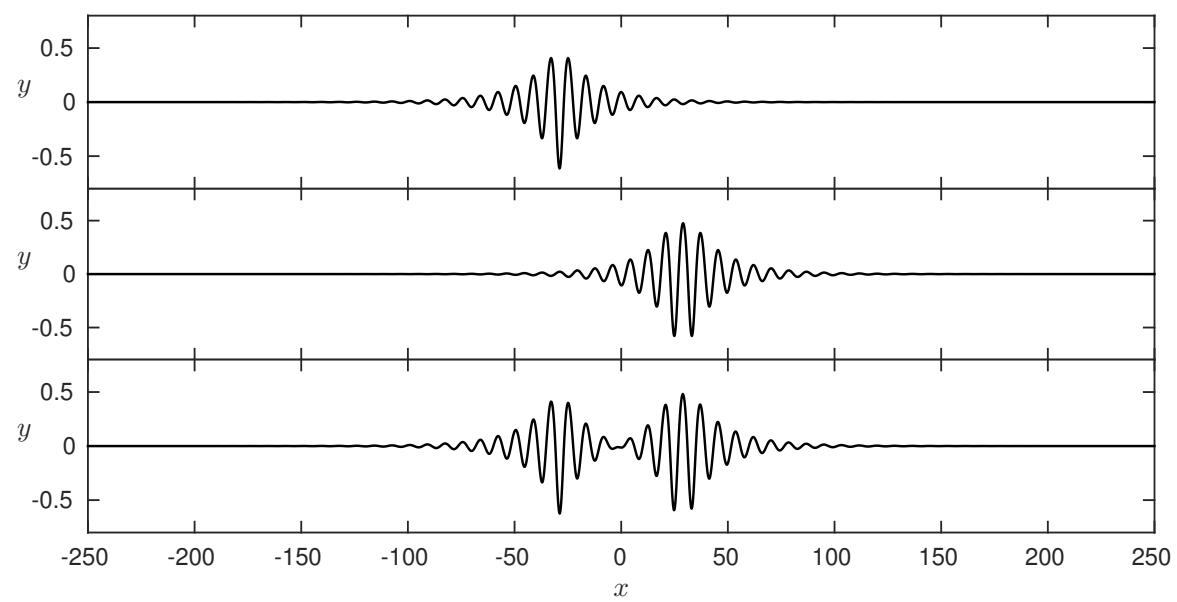

FIgURE 2. An initial guess for the computation of an asymmetric hydroelastic solitary-wave profile (bottom figure) which is composed of a depression wave (top figure) and a one-hump elevation wave (middle figure) both propagating at $c=1.32$.

generalised to hydroelastic waves without any essential modification due to the Hamiltonian structure (2.9). Furthermore, since all the disturbances are superharmonic for solitary waves, Saffman's conclusion is extremely useful for our problem. In $\S 5$, we will focus on the stability properties for solitary waves via direct numerical simulations together with Saffman's result. In figure 1(d)(e), the total energy is partitioned into three parts: kinetic energy (solid line), gravitational potential energy (dotted line), and elastic potential energy (dash-dotted line). We note that for the elevation branch different types of energies are similar in their behaviour but differ quantitatively, namely, for an elevation solitary wave, kinetic energy > gravitational potential energy > elastic potential energy (see figure 1(d) from top to bottom). For large-amplitude depression solitary waves (figure $1(\mathrm{e})$ ), as the translating speed decreases, the kinetic energy decreases and vanishes at $c=0$, while the elastic potential energy increases and reaches its global maximum at the static state.

\subsection{Asymmetric Waves}

Asymmetric gravity-capillary waves in deep water were recently computed by Wang et al. (2014) for the full Euler equations. Their existence is related to the possibility of having several symmetric waves travelling at the same speed. Figure 1(a) shows that for values of $c$ close to 1.3247 , we also have several symmetric flexural-gravity waves travelling at the same speed. This suggests to search for asymmetric waves for the present problem. In this section we provide numerical evidence that such waves do exist.

In order to compute asymmetric hydroelastic solitary waves, it is essential to choose a good initial guess for the Newton's iterations. Following the procedure described in Wang et al. (2014), we choose a depression wave (see the top graph in figure 2) and an elevation wave (see the middle graph in figure 2) computed in the last section. These two waves are chosen so that they travel at the same speed $c=1.32$ (this choice is possible for values of $c$ close to the minimum phase speed). We shift the profiles and then merge them in the middle as a new wave profile (see the bottom graph in figure 2). This new profile is considered as the initial guess of Newton's method and the algorithm converges 


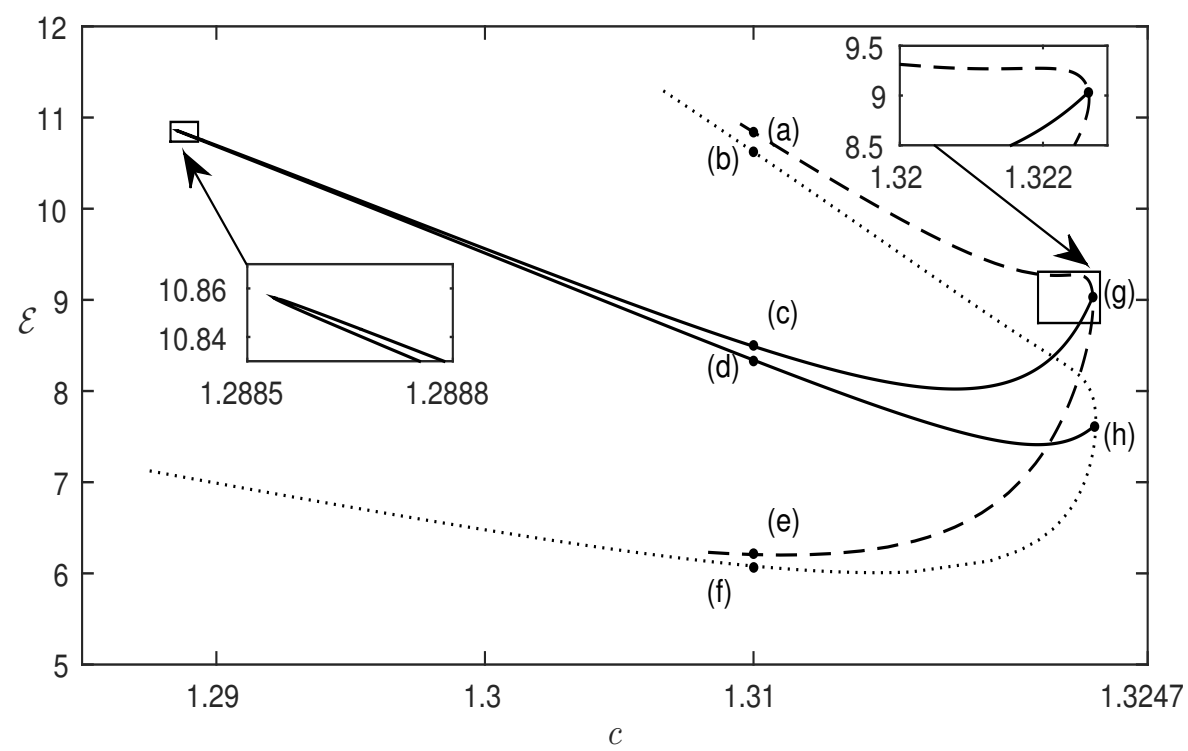

FiguRE 3. Speed-energy bifurcation diagram of asymmetric hydroelastic solitary waves. The solid curve correspond to the branch of asymmetric waves. The dotted curve and the dashed curve correspond to two branches of symmetric waves where the asymmetric branch bifurcates from. (a)-(f) correspond to those waves travelling at speed $c=1.31$ whose profiles are shown in figure 4.

to a solution after several iterations. After obtaining a convergent solution, we can follow the branch by using a continuation method. This yields the speed-energy bifurcation diagram shown in figure 3 .

We present in figure 3 values of the energy of asymmetric waves versus the speed $c$. The solid curve corresponds to asymmetric waves, whereas the dotted curve and the dashed curve correspond to symmetric waves. Typical wave profiles corresponding to the points labelled (a)-(h) are shown in figure 4 including two asymmetric ones (figures $4(\mathrm{c}, \mathrm{d}))$. The curves of figure 3 show that the speed of asymmetric solitary waves is always below the minimum of the phase speed therefore these waves do not resonate with linear periodic waves, namely, they do not turn into generalised solitary waves or into periodic waves (see Milewski et al. (2011) for a discussion). As can be seen from figure 3, the solid branches join the dashed and the dotted branches, i.e. the asymmetric waves eventually become symmetric (see profiles $(\mathrm{g})$ and $(\mathrm{h})$ in figure 4 ). It shows that the asymmetric waves appear from a spontaneous symmetry-breaking bifurcation and vanish at another symmetry-breaking bifurcation. It is worth mentioning that profiles $4(\mathrm{a}, \mathrm{b})$ from the upper branches are essentially consisting of two identical elevation waves, while the profiles $4(\mathrm{e}, \mathrm{f})$ from the lower branches are two depression waves merged at the origin. Energywise, the results are quite reasonable since an elevation wave possesses more energy than a depression wave with the same speed.

There exist many other families of asymmetric solitary-wave solutions which can be found by using different initial guesses because of the many possible choices of elevation waves travelling at the same speed as demonstrated in figure 1 . We leave it as a future research interest and focus on the stability problem. 

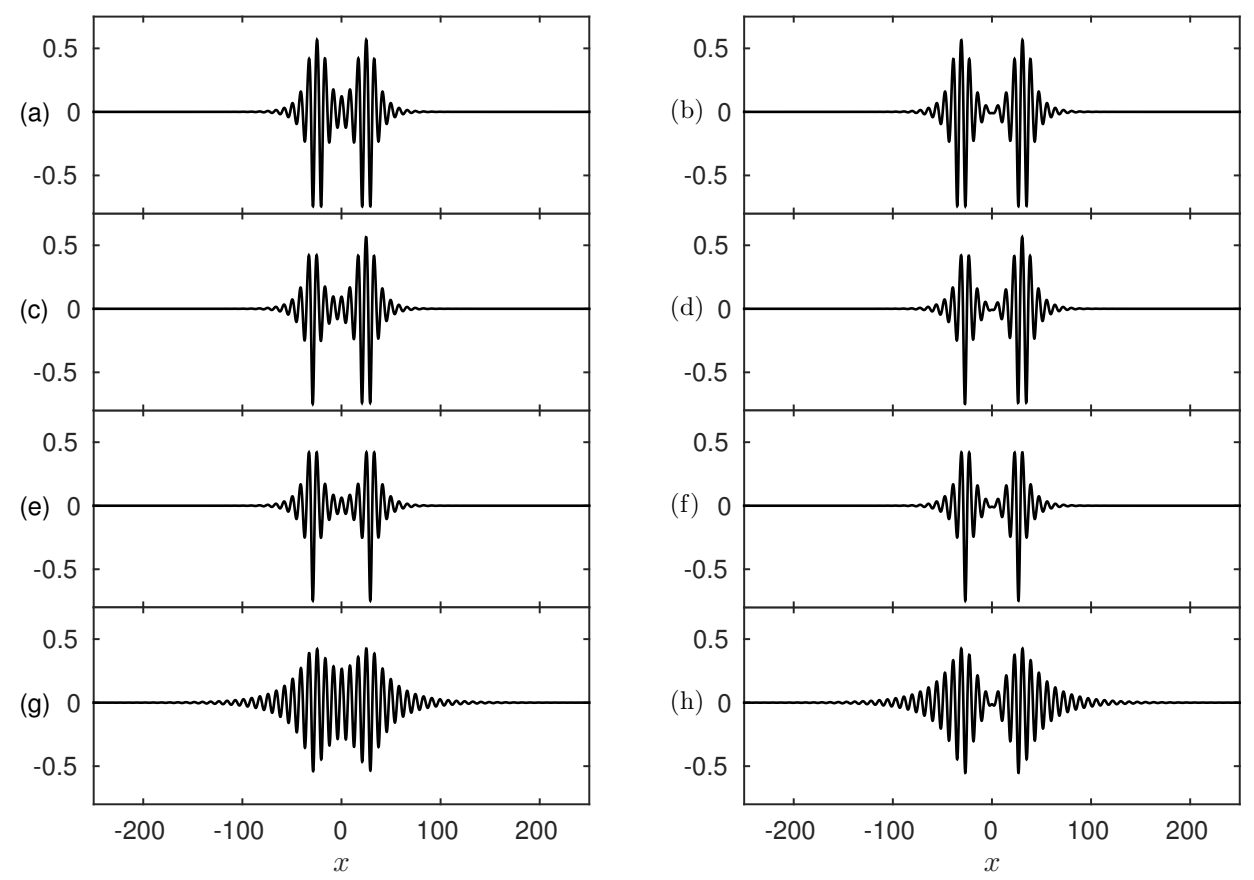

Figure 4. Typical profiles correspond to points (a)-(h) indicated in figure 3. (c) and (d) are the typical profiles of asymmetric waves; $(\mathrm{g})$ and $(\mathrm{h})$ correspond to the symmetry-breaking bifurcation points. All figures are shown in the physical space.

\section{Stability of Solitary Waves}

In this section, we investigate the stability of the waves computed in $\S 4$ when they are subjected to longitudinal perturbations. This is achieved by solving numerically the evolution equations (3.5) and (3.6) with the (slightly perturbed) travelling wave as an initial condition. Both symmetric and asymmetric waves are considered. For convenience we choose a frame of reference moving with the speed of the unperturbed solitary wave.

\subsection{Symmetric waves}

We start with the symmetric depression solitary waves. The speed-energy bifurcation curve for the depression branch shown again in figure 5 has two stationary points labelled (1) and (2). According to Saffman (1985), stability exchanges may occur at these points. We first investigate the stability of the waves before the first stationary point $(c \lesssim 0.8)$. The waves in this regime are large and steep, and some are even overhanging. Therefore the numerics becomes very stiff if an explicit time integration method is used because of the strong constraint on the time step. We remove this constraint by using the backward Euler's method which is implicit. We take a large-amplitude depression solitary waves $\eta(\xi)$ with $c=0.5$ and $\eta(0)=-2.70$, which is overhanging (see the top figure of the right panel of figure 6). At $t=0$, a small perturbation $0.01 \cos (\xi) \eta(\xi)$ is added to the steady solution. The system (3.5) and (3.6) is then integrated by the backward Euler's method with the time step $d t=0.002$, and at each step the discretised nonlinear algebraic system is solved by Newton's method. The time evolution of the perturbed wave is shown in the left part of figure 6 . The profiles in the physical space at time $t=0,2,4$ are presented in 


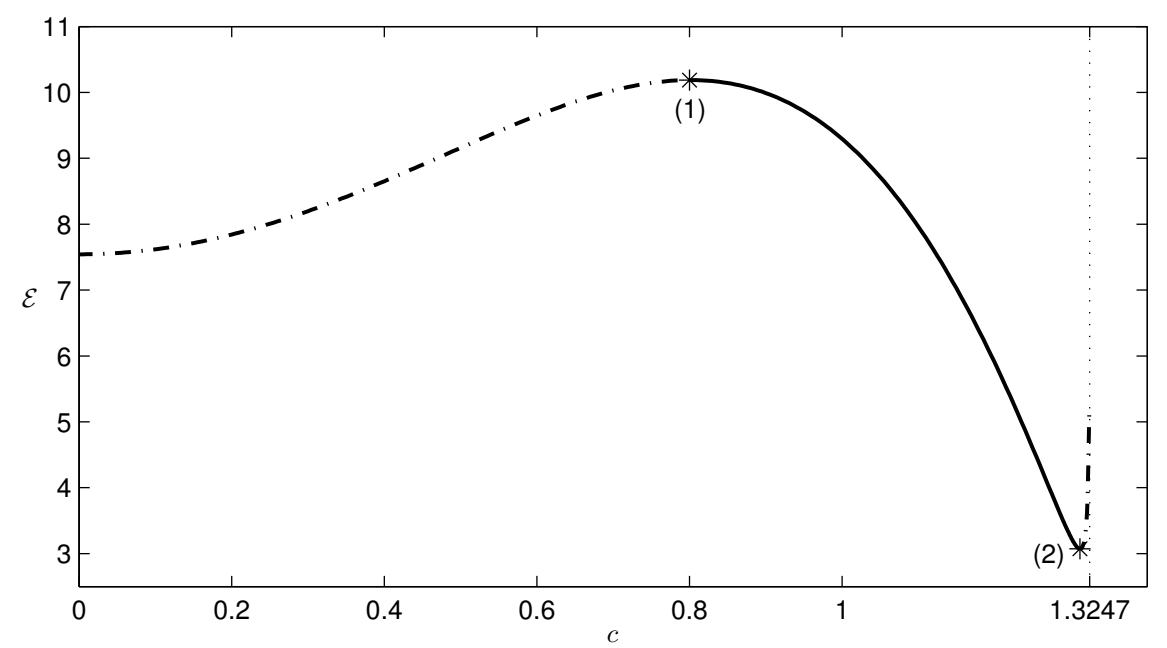

FIGURE 5. Stability of the depression branch. Waves from the solid segment are stable whereas those from the dash-dotted parts are unstable. The stationary points have been marked as stars in the graph.
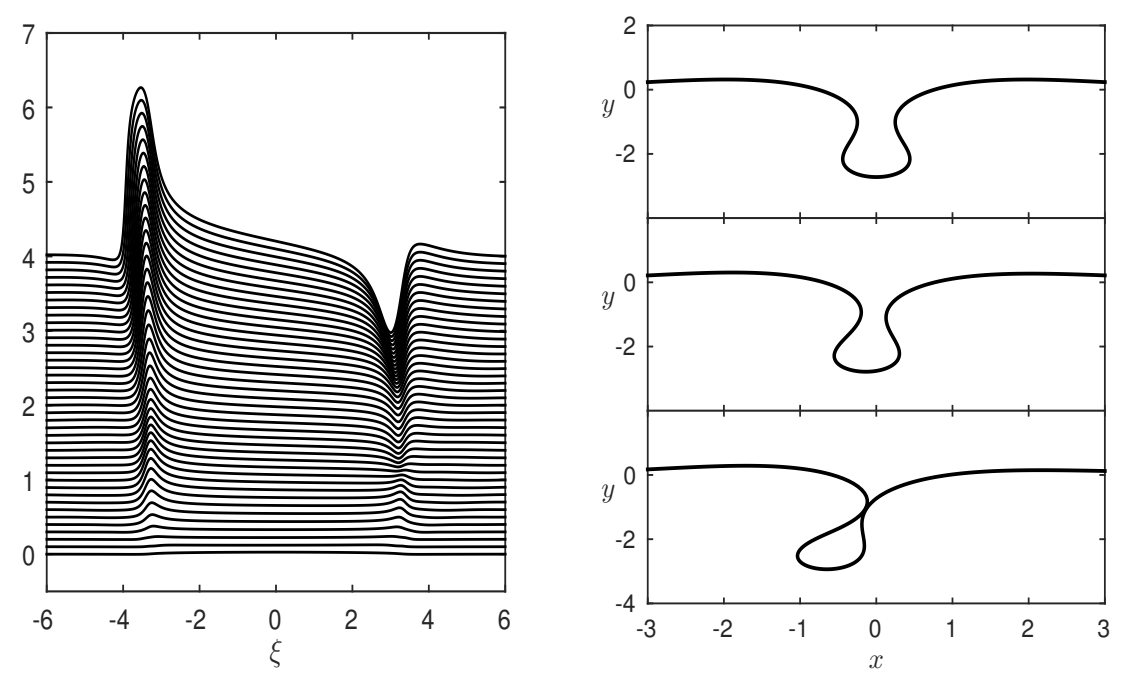

Figure 6 . Time evolution of a large-amplitude depression solitary wave propagating with the velocity $c=0.5$ and amplitude $\eta(0)=-2.70$. The computation was performed with $d t=0.002$ and $d \xi=0.01$. Left: time evolution of the perturbation which is initially $0.01 \cos (\xi) \eta(\xi)$. Right: wave profiles in the physical space at time $t=0,2,4$ (right top to bottom). Only the main parts of the solutions are shown in figures.

the right panel from top to bottom. We can conclude that the bubble changes its shape quickly and then becomes unstable. We stop the computation when the profile touches itself forming a closed bubble. We can further deduce from this numerical experiment that the depression solitary waves lie in the segment $0<c \lesssim 0.8$ are all unstable according to Saffman's theory.

For the remaining time-evolution computations, we use a fourth-order Runge-Kutta method rather than the backward Euler's scheme since the problems are not extremely 

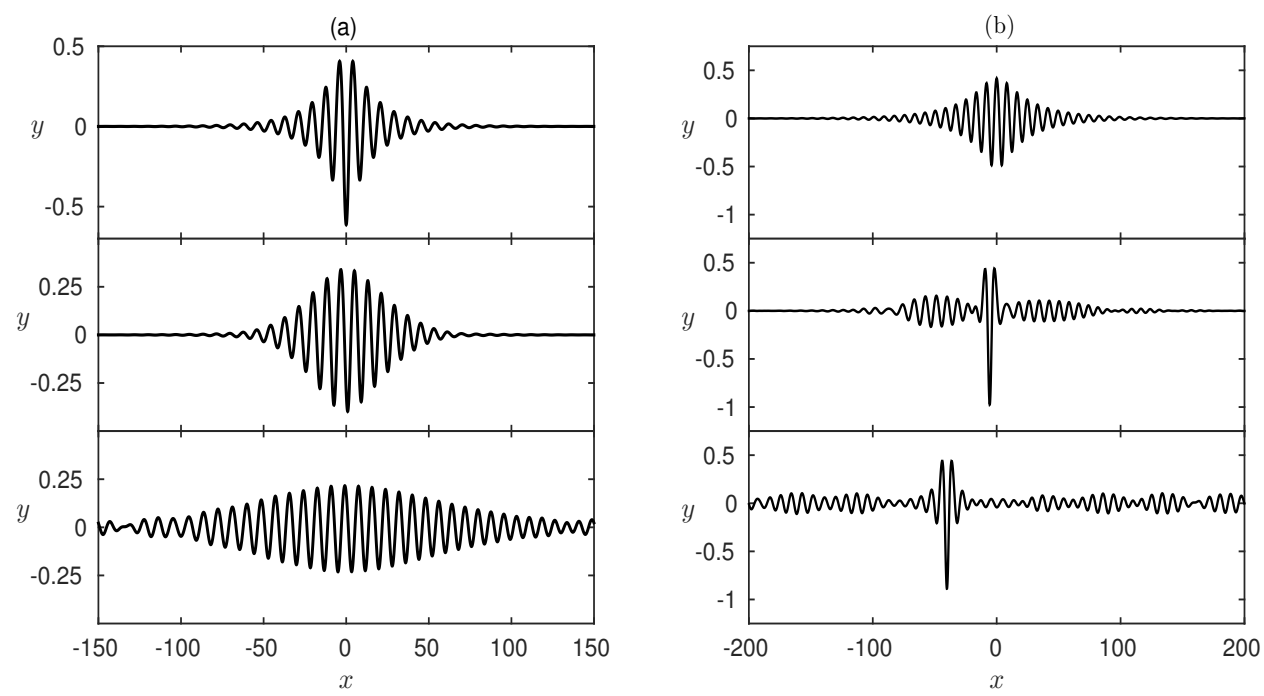

Figure 7. Time-evolution of (a) a perturbed depression wave $(c=1.32)$ at $t=0,1000,2000$ (from top to bottom); (b) a perturbed elevation wave $(c=1.323$ ) at $t=0,1500,3000$ (from top to bottom).

stiff for moderate-amplitude waves. In most of the computations the mesh size in space and the step size in time are chosen as $d \xi=0.1$ and $d t=5 \times 10^{-4}$ respectively.

For the solid part in figure 5 (the segment between the stationary points (1) and (2)), a variety of perturbations with $5 \%$ of the energy of the initial depression solitary waves did not show instability. However, as the speed increases and passes the second stationary point (2), the solitary wave becomes unstable again. The snapshots of the dynamics of a depression solitary wave in this region subject to the $1 \%$ amplitude-decreasing perturbation are shown in figure $7(\mathrm{a})$. The wave eventually disperses out as time evolves. Finally we can draw the conclusion that for the depression branch of hydroelastic solitary waves, there are two stationary points in the speed-energy bifurcation diagram and that the stability exchanges occur at both of them.

The stability problem for the elevation branch is more complicated since the speedenergy curve has many stationary points and turning points, and we need to choose one typical example between every two successive critical points and check its stability. In the speed-energy bifurcation diagram (figure 8), the stationary points and the turning points have been numbered from (1) to (7). We found that only the waves between the turning point (2) and stationary point (3) (solid curve in figure 8) are stable when subjected to longitudinal perturbations. All other waves on the elevation branch (dot-dashed curve in figure 8) turn out to be unstable.

We present some time-evolutions of elevation waves to demonstrate their stability properties. A wave from the right of point (1) has been perturbed by $1 \%$ of the amplitude of the steady wave. The snapshots show a little focussing phenomenon and a symmetrybreaking instability as well, and finally become a stable depression solitary waves with a radiated wave field of linear wave packets shedding on both sides (see figure 7(b)). Figure 9 (a) displays a stable elevation wave which is located between point (2) and (3) of figure 8 . These stable elevation solitary waves feature two big troughs separated by a small dimple. A 5\% amplitude-decreasing perturbation has been applied to the initial solitary wave but did not show any instability for a long-time computation. It is worth mentioning that the amplitude-decreasing perturbation results in a wave of slightly smaller energy but 

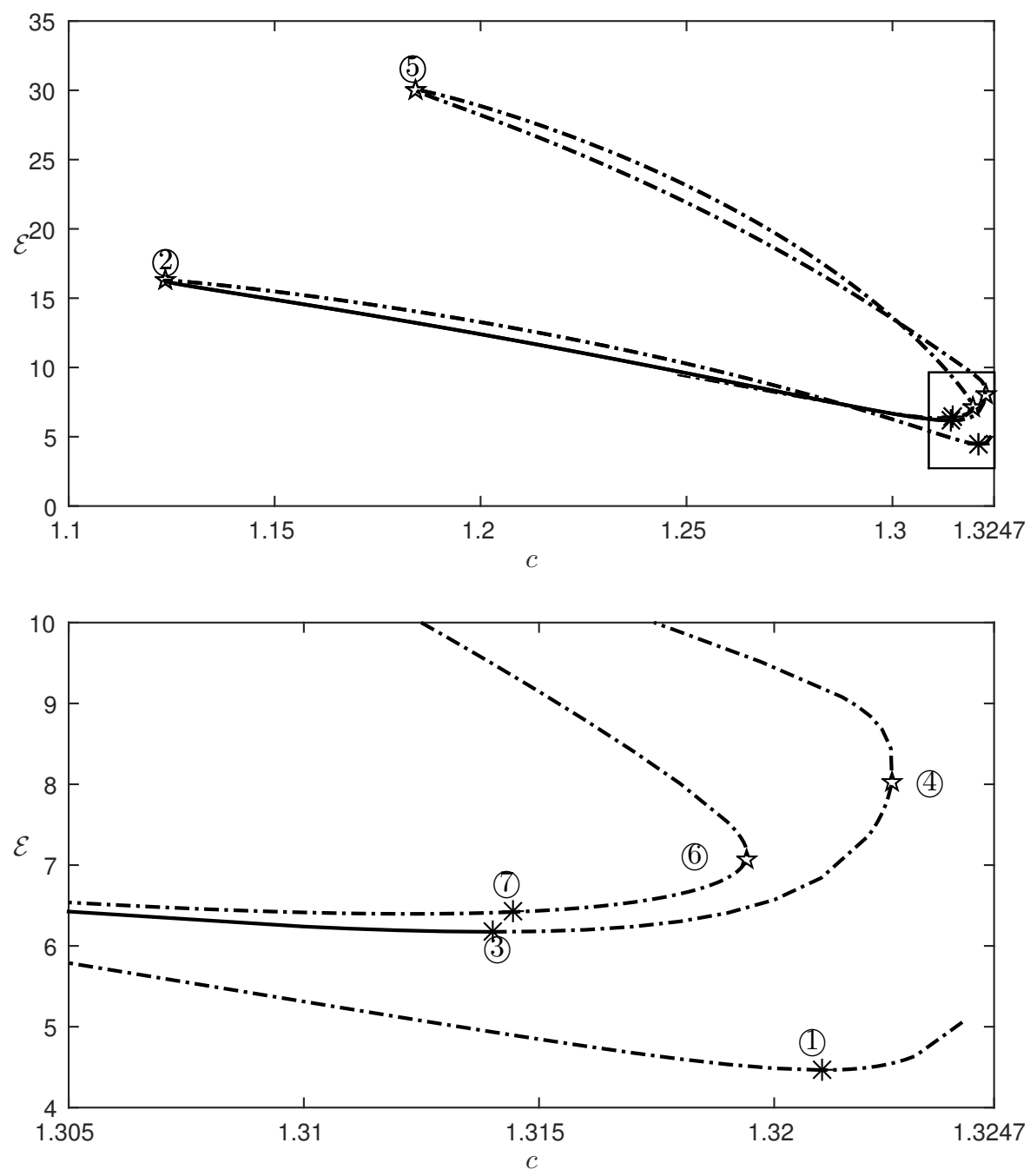

FIGURE 8. Speed-energy bifurcation curve for the branch of elevation. The graph on the bottom is a zoom in of the box in the graph on the top. Waves from the solid branches are stable whereas those from the dot-dashed curves are unstable. The stationary points and the turning points are marked as stars and pentagrams respectively in the graphs.

larger speed than the original one which causes the right translation in the moving frame. However, not all the elevation waves featuring two depression solitary waves placed sideby-side are stable. Figure 9 (b) presents an example of unstable elevation waves composed by two troughs with a small hump. The wave is located on the very last branch, i.e. beyond (7), with $c=1.25, \eta(0)=0.113$ and $\mathcal{E}=9.34$. We choose a specific perturbation which decreases the amplitude of the left trough by $0.5 \%$ and increases the right one by $0.5 \%$. Therefore the speed of the left trough is greater than that of the right trough so that an overtaking collision occurs from which we can deduce the instability.

\subsection{Asymmetric Waves}

We now move on to the stability of the asymmetric solitary waves. It turns out that the whole branch computed in $\S 4.2$ is unstable. One typical example is shown in figure 10, 

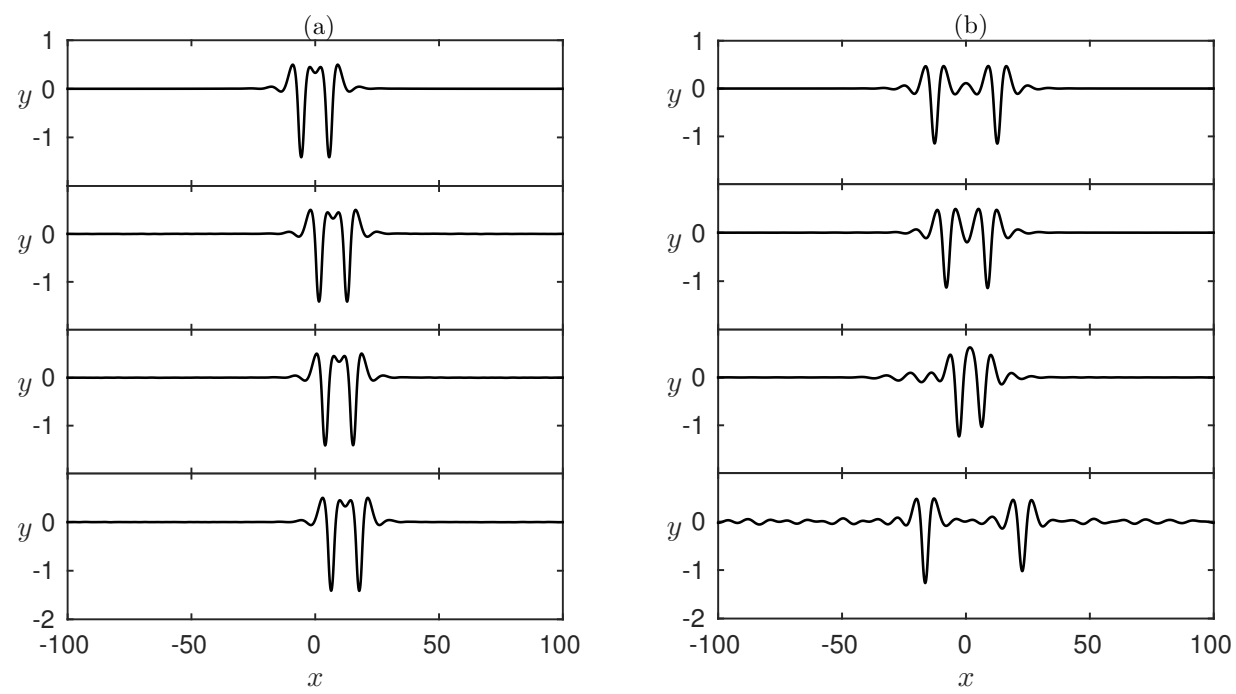

FiguRE 9. Time-evolution of (a) a perturbed elevation wave $(c=1.2)$ at $t=0,3000,4000,5000$ (from top to bottom); (b) a perturbed elevation wave $(c=1.25)$ at $t=0,750,1000,1500$ (from top to bottom).

where we take an asymmetric solitary wave featuring a depression wave and an elevation waves connected with small ripples as the initial data (figure 10a). As time goes on, the left single trough remains unchanged but the elevation part on the right abruptly turns to be one big trough (figure 10b). The right trough travels leftwards since its speed is less than the speed of the moving frame (figure 10c). Therefore the stability problem becomes an overtaking collision and only one big trough survives (figure 10d). Numerical experiments have been performed for other asymmetric solitary waves on the same branch. These were unstable in all cases tried.

\section{Dynamics of the Solitary Waves}

In this section we supplement the stability analysis of $\S 5$ by studying the interaction of solitary waves and their generation by moving disturbances.

\subsection{Collision}

We first consider head-on collisions and overtaking collisions between stable hydroelastic solitary waves. In all the collision experiments, the initial data were constructed by first shifting the existing travelling waves so as to minimise their overlap, and then adding them together.

A typical example of head-on collisions is shown in figure 11. Here a stable elevation wave travelling rightwards collides with a stable depression one travelling leftwards. The collision is inelastic even though both waves survive after the collision, since a lot of visible ripples shed on both sides during the interaction. Several other computations of head-on collisions were carried out, and in all cases the collisions appeared to be inelastic.

The second set of numerical experiments on the dynamics of hydroelastic solitary waves is of overtaking collisions. We choose two stable depression waves travelling in the same direction but with different translating speeds. When the amplitude difference between the two is large, only one wave survives after the collision with a radiation field. However, 
(a)

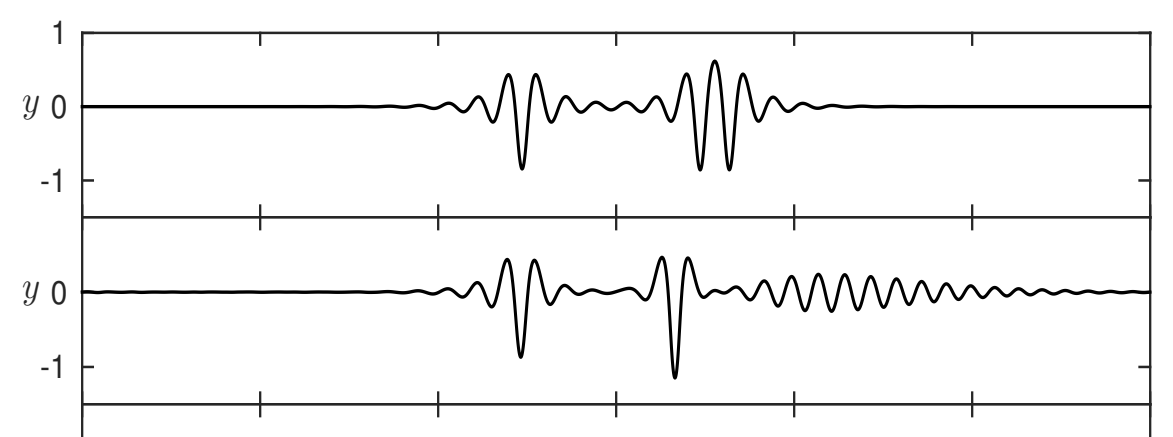

(c)

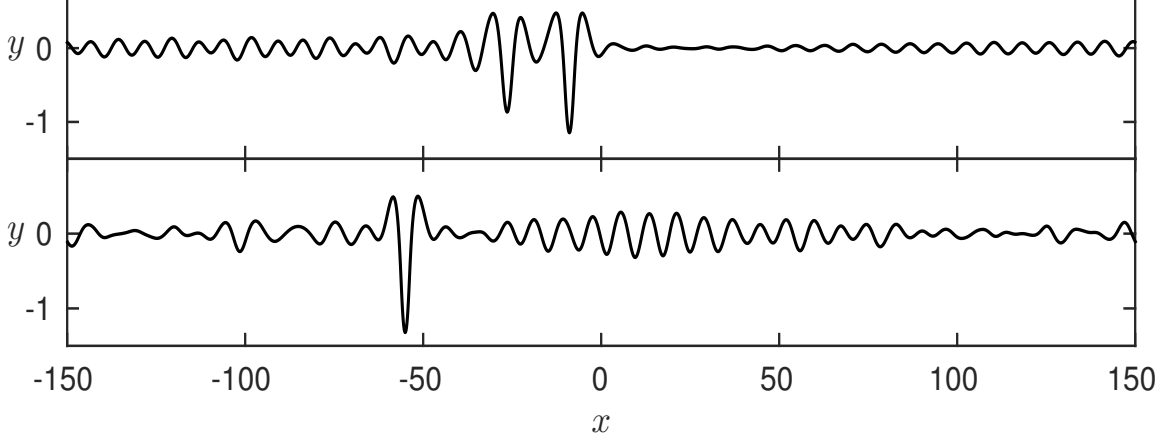

FiguRE 10. Time evolution of an asymmetric solitary wave $(c=1.3)$ perturbed by numerical noises. Snapshots are shown for $t=0,250,750,1250$ (from top to bottom).

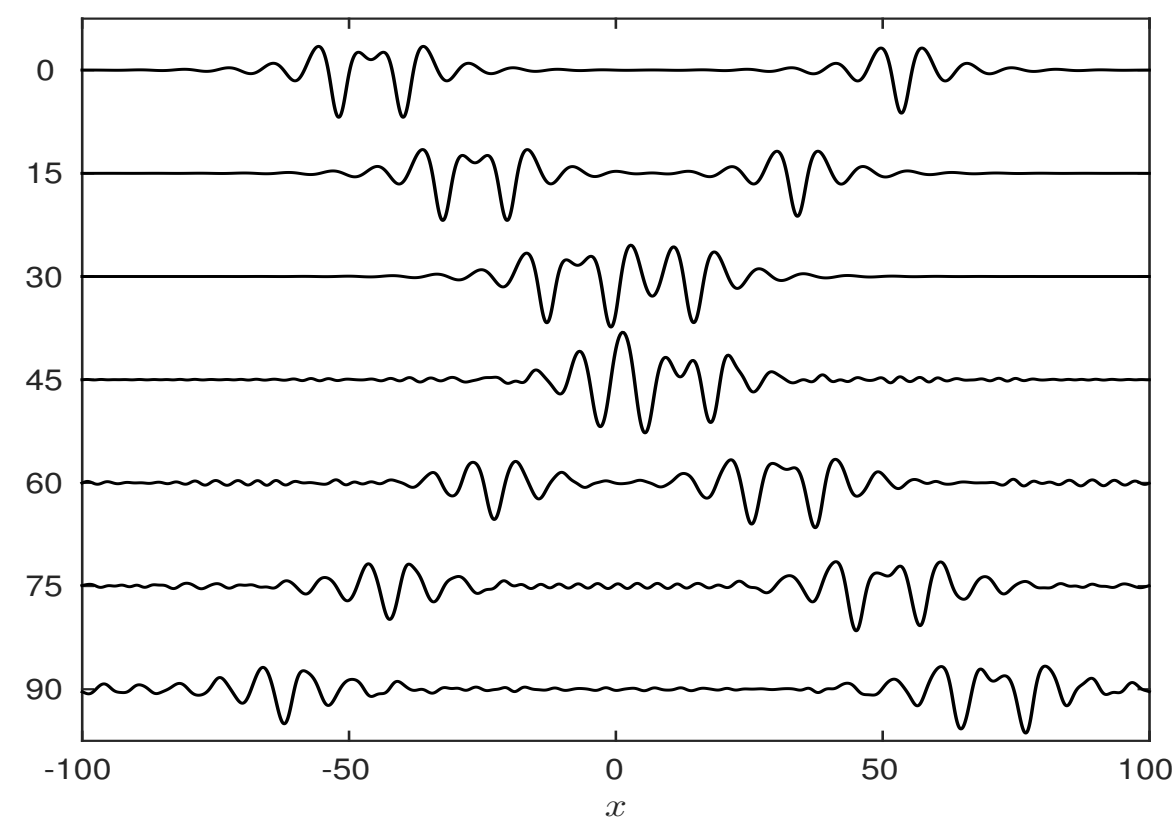

FiguRE 11. Head-on collision. An elevation wave travels rightwards at $c=1.3$ and a depression wave travels leftwards at $c=1.3$. We plot $y$ versus $x$ for time $t=0,15,30,45,60,75,90$ (from top to bottom). 
both depression wave can survive if their amplitude difference is small. As can be seen from figure 12(a), the overtaking collision is also inelastic when both waves survive since some radiation field is generated during the interaction. The error, which is of order $10^{-12}$ through the whole computation, shows that the energy is conserved to a high accuracy. The difference between the initial data and the solution obtained by reversing time after $t=4200$ in the collision is of order $10^{-9}$ (see figure $12 \mathrm{~b}$ ). This further validates the time-dependent codes. We can also choose a stable elevation wave to overtake a depression one, and vice versa. One example of this kind of collision is presented in figure 13. The structure of the elevation solitary wave with two well-separated troughs was destroyed during the interaction, and two depression waves travelling at different speeds were ultimately observed.

\subsection{Excitation}

The coupling between a moving load and the hydroelastic waves generated has attracted a long interest because its applications in polar regions (Wilson (1958); Takizawa (1985); Squire et al. (1988)). According to the linear theory, the critical velocity for resonance between the moving load and the hydroelastic waves occurs at the minimum of the phase speed. The resonance results in an accumulation of energy and an unlimited growth of the elastic-sheet displacement. Therefore the linear theory fails to predict the physical behaviour of the system and a nonlinear theory needs to be developed. This was considered by Părău \& Dias (2002); Bonnefoy et al. (2009); Milewski et al. (2011) for the Kirchhoff-Love model and by Guyenne \& Părău $(2012,2014)$ for Toland's model with reduced equations. The fundamental phenomena of the nonlinear problem is the shedding of solitary waves. Here we examine the problem by using the full Euler equations and Toland's model. A single moving pressure which is defined as

$$
P=A e^{-(x+220-c t)^{2} / 16},
$$

is applied in (3.6) initially with the amplitude $A=0.2$ and a translating speed $c=1.3$. The pressure is switched off at $t=125$. We found that the solution rapidly relaxes to a symmetric depression wave after the forcing is released (figure 14). The stability of this large-amplitude, fully-localised response is confirmed from its steady propagation at long times.

The stability analysis in $\S 5$ shows that elevation waves with the structure of two wellseparated troughs connected by a dimple are also longitudinally stable. We now show that these stable elevation waves can also be generated by moving loads. We choose the load as two fully-localised pressures moving simultaneously on the ice sheet at the speed $c$. These pressures are defined as

$$
\begin{aligned}
& P_{1}=A e^{-(x+220-c t)^{2} / 16}, \\
& P_{2}=A e^{-(x+220-d-c t)^{2} / 16},
\end{aligned}
$$

where $A$ is a parameter measuring the magnitude of the pressures and $d$ is the distance between the centres of two pressures. Figure 15 shows the snapshots of the generation of a stable elevation solitary wave by two pressures moving with the same speed. An elevation wave of two big troughs quickly forms after the forcing is turned off (the 3rd and 4th pictures in Figure 15). From longer computations (not shown), we believe that these solitary waves are stable and robust. This large response can propagate without changing its main structure, inspite of the interactions with the background radiation induced by the initial generation process. This numerical experiment can be used to mimic 
(a)

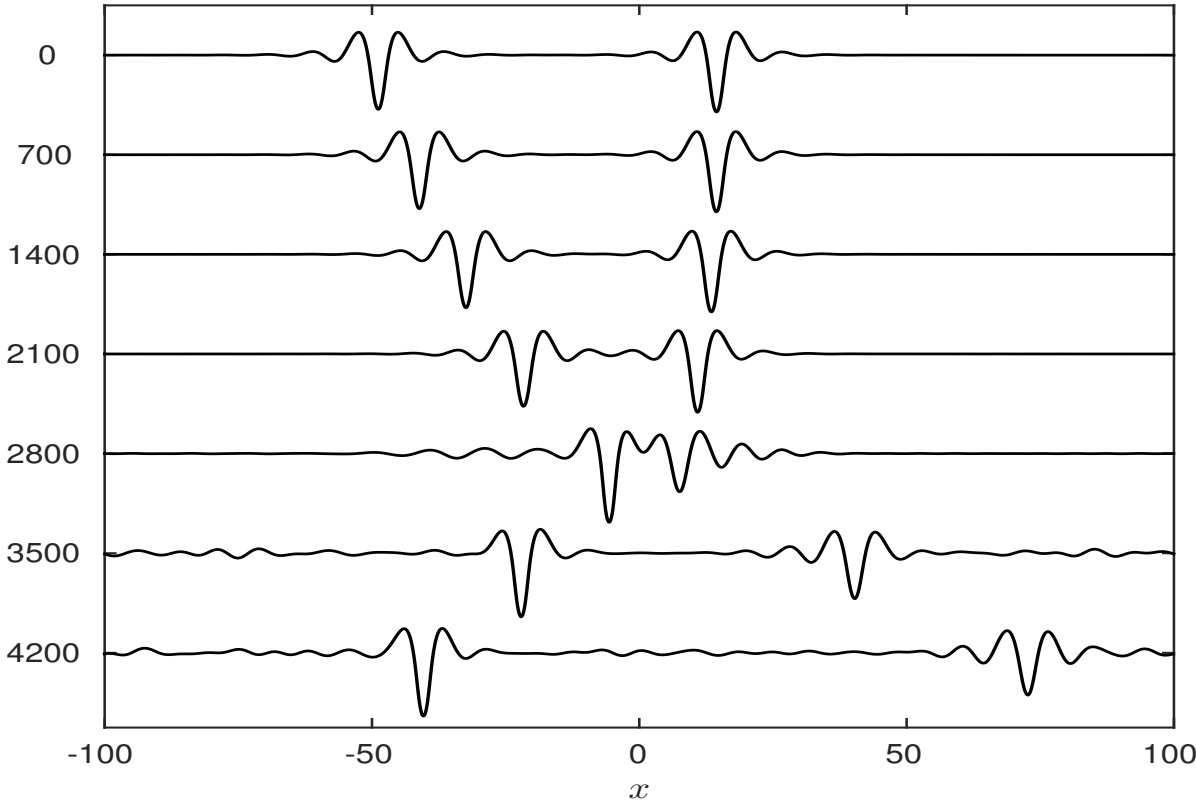

(b)

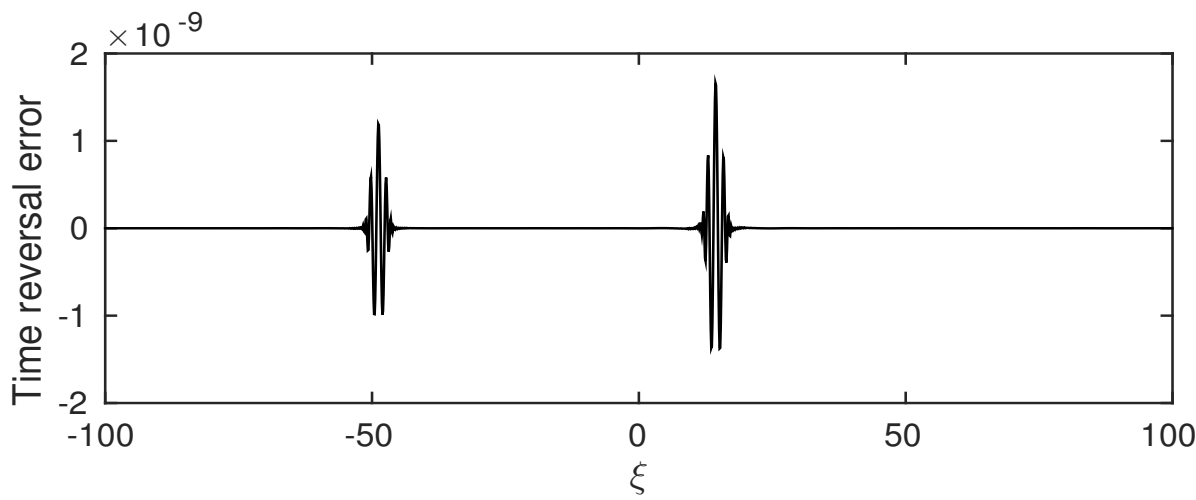

FiguRE 12. Overtaking collision. Two depression waves travel rightforwards at $c=1.26$ and $c=1.25$ respectively. (a) We plot $y$ versus $x$ for time $t=0,700,1400,2100,2800,3500,4200$ (from top to bottom). The snapshots are shown in a frame of reference moving with the speed $c=1.25$. (b) The difference between the initial data and the time-reversed solution computed from $t=4200$ back to $t=0$.

cars or aircrafts running successively on a very large floating structure and suggests that fully-localised two-trough structure may be observable in a real situation.

\section{Conclusions}

Two-dimensional hydroelastic solitary waves propagating in deep water were investigated. The nonlinear model proposed by Toland (2008) was used to formulate the pressure exerted by the thin elastic sheet. There are depression and elevation solitary waves which are symmetric in the direction of wave propagation. These two branches, which were previously computed and extended respectively by Guyenne \& Părău (2012) and Wang et al. (2013), were quickly reviewed. The depression and the elevation branches 


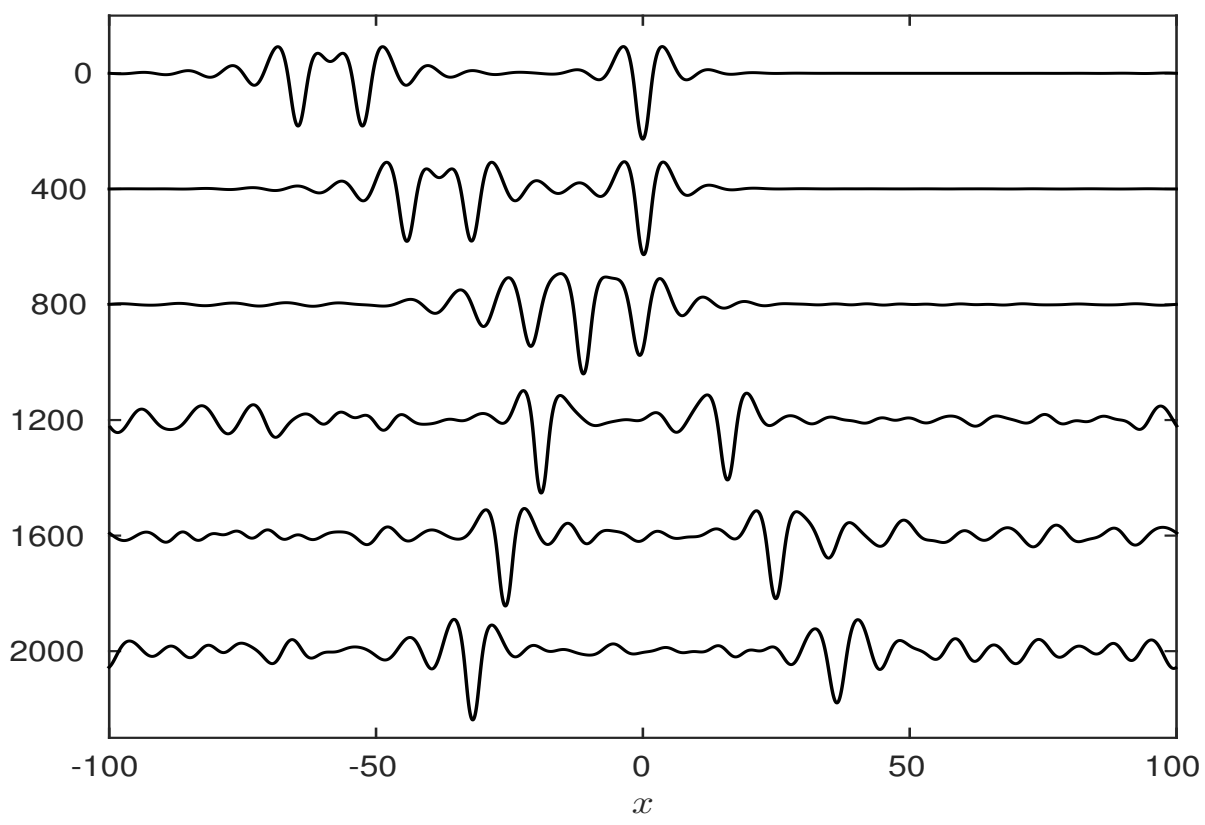

Figure 13. Overtaking collision. An elevation wave travels rightforwards at $c=1.3$ and a depression travels at $c=1.25$ in the same direction. We plot $y$ versus $x$ for time $t=0,400,800,1200,1600,2000$ (from top to bottom). The snapshots are shown in a frame of reference moving with the speed $c=1.25$.

bifurcate from the minimum of the phase speed and exist at subcritical speeds. The fact that different symmetric solitary waves can propagate at the same speed inspires us to search for asymmetric hydroelastic solitary waves. We superposed a depression wave and an elevation one as the initial guess to find an asymmetric solitary wave by using Newton's method. The complete bifurcation diagram was then constructed by a numerical continuation method. These asymmetric waves have a two-packet structure and exist only in the subcritical regime. The asymmetric branch appears from a spontaneous symmetry-breaking bifurcation and end up with another symmetry-breaking bifurcation.

The stability properties of all the hydroealstic solitary waves found to date, subject to longitudinal perturbations were studied systematically by using the time-dependent conformal map technique together with a backward Euler's method or a fourth-order Runge-Kutta method to integrate the full Euler equations in time. Our numerical experiments show that moderate-amplitude depression waves (lying between the stationary points (1) and (2) in figure 5) and certain elevation waves with two troughs connected by a dimple (lying in the segment between the turning point (2) and the stationary point (3) in figure 7) are stable. These two kinds of stable solitary waves can both be excited by applying one or two loads on the elastic sheet and moving the loads with a constant speed slightly below the phase speed minimum. Once the system gains enough localised energy, a stable hydroelasitc solitary wave with the radiation field can be obtained after the pressure is switched off.

There are other possible fluid system with complicated solitary-wave branches whose instability is worth investigating, most notably the interfacial gravity-capillary waves between two immiscible fluids. The results of Laget \& Dias (1997) show that if ratio of 


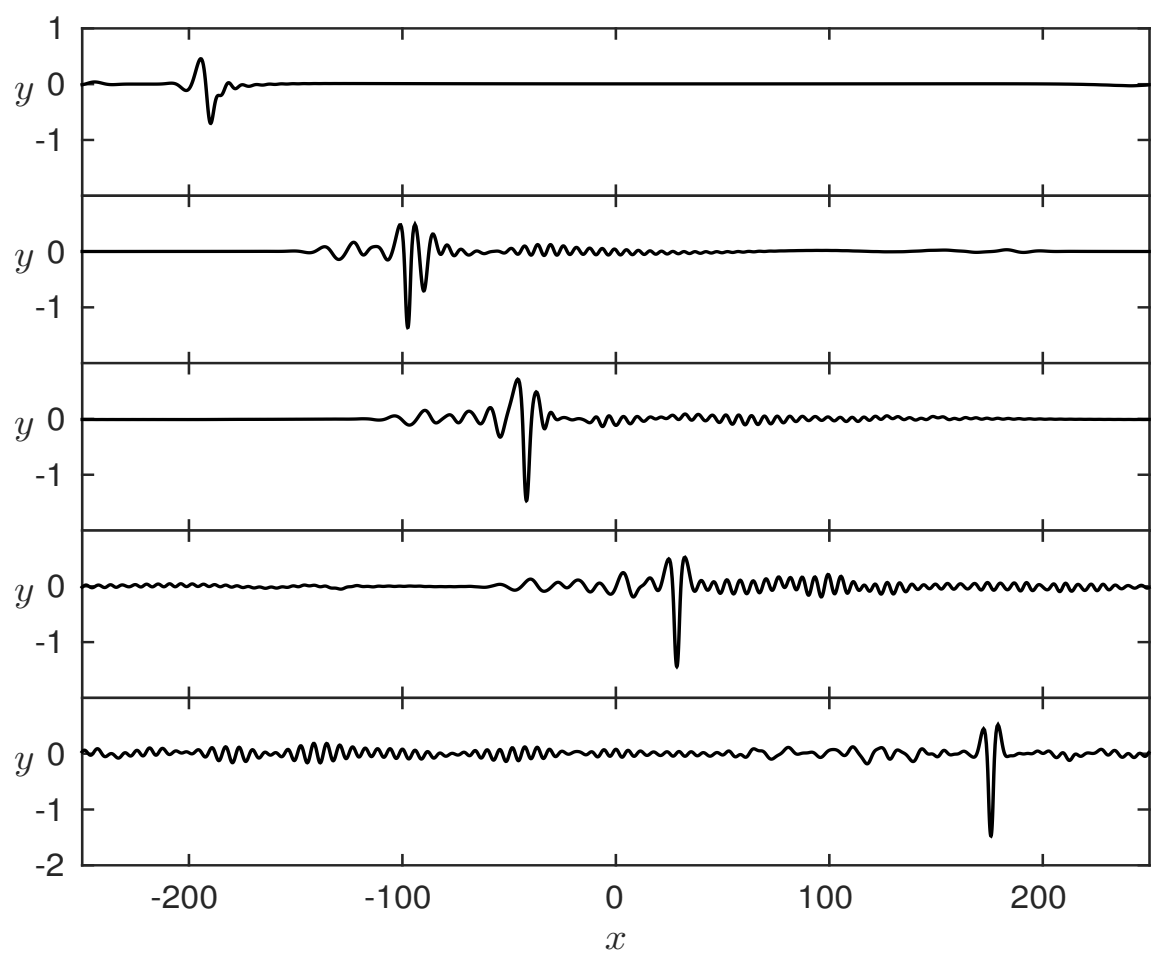

Figure 14. Snapshots of the free-surface due to large-forcing moving pressures $(A=0.2$ and $u=1.3$ ) at $t=25,100,140,200,325$ (from top to bottom). The forces is switched on at $t=0$ and off at $t=125$. It is initially placed at $x=-220$.

the density of the upper fluid to that of the lower fluid is larger than a critical value, the bifurcation diagram (figure 14 in their paper) is similar to figure 1. Therefore a study of the stability of these interfacial solitary waves is of interest. It is also noted that the stability of multi-packet solitary waves was studied by Chardard et al. $(2009,2011)$ using the Maslov theory. One might ask whether or not this theory can be applied to the hydroelastic solitary waves.

We remark that we have considered in the present paper an idealised model which includes only the bending (i.e. beam-like response) of the material in order to identify the main properties of the waves. Other effects such as the stretching (i.e. rubber-like response), mass, thickness and inertia of the material can be considered in further studies. We finally comment that though the overhanging waves are theoretically plausible in the idealised model, in practice the bending of the sheet will cause cracking for some critical curvature, and overturning of a finite mass sheet must cause the sheet to fall off the fluid.

Acknowledgment This work was supported by EPSRC, under grant no. EP/J019569/1.

\section{REFERENCES}

Bonnefoy, F., Meylan, M. H. \& Ferrant, P. 2009 Nonlinear higher-order spectral solution for a two-dimensional moving load on ice. J. Fluid Mech. 621, 215-242. 


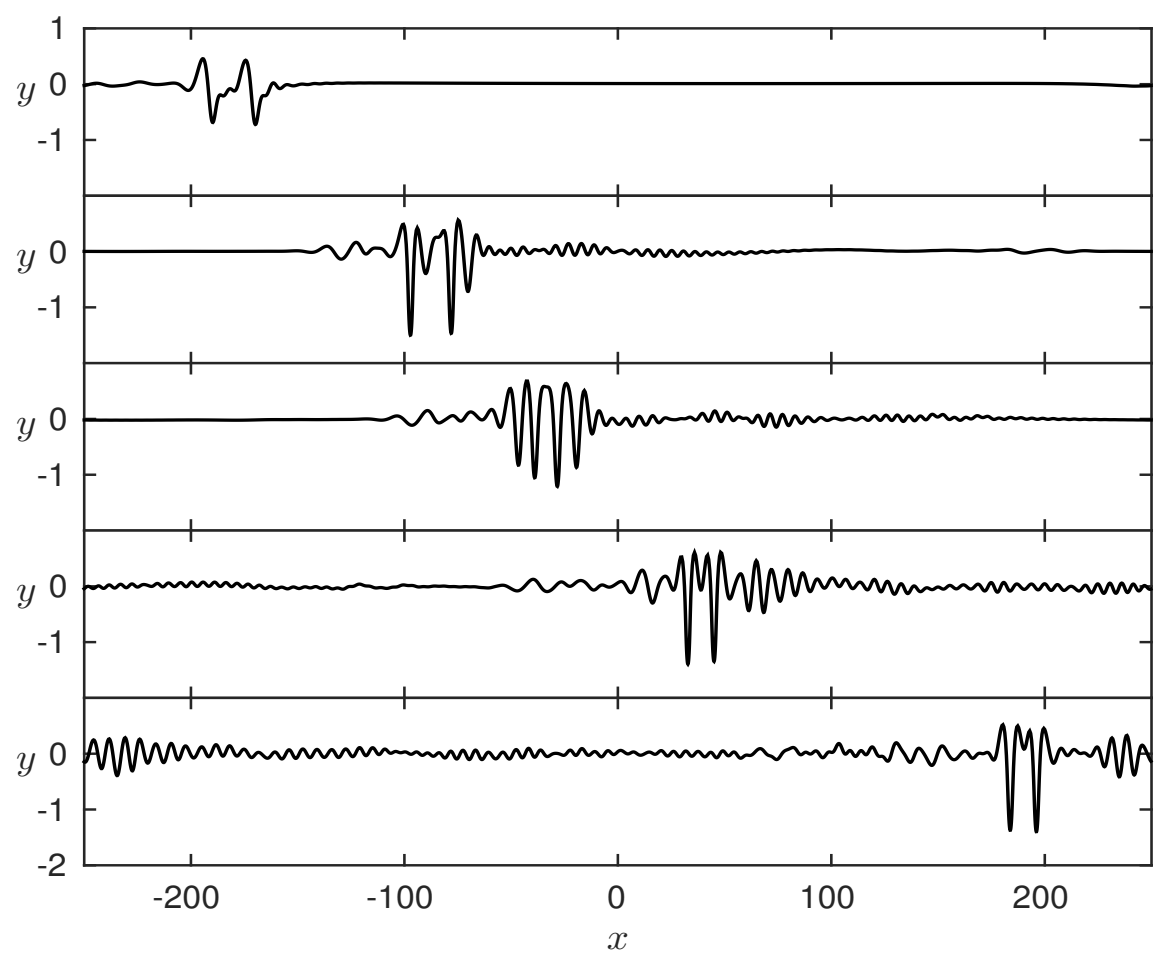

Figure 15. Snapshots of the free-surface due to two moving pressures $(A=0.2$ and $c=1.3$ ) at $t=25,100,140,200,325$ (from top to bottom). The forces are switched on at $t=0$ and off at $t=125$. The centre of $P_{1}$ is initially placed at $x=-220$ and that of $P_{2}$ is at $x=-200$, i.e., $d=20$.

CRAPper, G. D. 1957 An exact solution for progressive capillary waves of arbitrary amplitude. J. Fluid Mech. 2, 532-540.

Chardard, F., Dias, F. \& Bridges, T. J. 2009 Computing the Maslov index of solitary waves, Part 1: Hamiltonian systems on a four-dimensional phase space. Physica $D \mathbf{2 3 8}$, 1841-1867.

Chardard, F., Dias, F. \& Bridges, T. J. 2011 Computing the Maslov index of solitary waves, Part 2: Phase space with dimension greater than four. Physica D 240, 1334-1344.

Dyachenko, A. I., Kuznetsov, E. A., Spector, M. D. \& Zakharov, V. E. 1996 Analytical description of the free surface dynamics of an ideal fluid (canonical formalism and conformal mapping). Phys. Let. A 221, 73-79.

Forbes, L. K. 1986 Surface waves of large amplitude beneath an elastic sheet. I. High-order series solution. J. Fluid Mech. 169, 409-428.

ForBes, L. K. 1988 Surface waves of large amplitude beneath an elastic sheet. part 2. galerkin solution. J. Fluid Mech. 188, 491-508.

Gao, T. \& Vanden-Broeck, J.-M. 2014 Numerical studies of two-dimensional hydroelastic periodic and generalised solitary waves. Phys. Fluids 26, 087101.

Greenhill, A. G. 1886 Wave motion in hydrodynamics. Amer. J. Math. 9, 62-96.

Guyenne, P. \& PĂRĂU, E. I. 2012 Computations of fully nonlinear hydroelastic solitary wave on deep water. J. Fluid Mech. 713, 307-329.

Guyenne, P. \& PĂRĂU, E. I. 2014 Finite-depth effects on solitary waves in a floating ice sheet. J. Fluids Struct. 49, 242-262.

JAIN, A. K. 1994 Review of flexible risers and articulated storage systems. Ocean Eng. 21, 733-750. 
LAget, O. \& DiAs, F. 1997 Numerical computation of capillary-gravity interfacial solitary waves. J. Fluid Mech. 349, 221-251.

Longuet-Higgins, M. S. 1988 Limiting forms for capillary-gravity waves. J. Fluid Mech. 194, $351-375$.

Marko, J. R. 2003 Observations and analyses of an intense waves-in-ice event in the Sea of Okhotsk. J. Geophys. Res. 108, 3296.

Milewski, P. A., Vanden-Broeck, J.-M. \& Wang, Z. 2010 Dynamics of steep twodimensional gravity-capillary solitary waves. J. Fluid Mech. 664, 466-477.

Milewski, P. A., VAnden-Broeck, J.-M. \& WANG, Z. 2011 Hydroelastic solitary waves in deep water. J. Fluid Mech. 679, 628-640.

Milewski, P. A. \& WAng, Z. 2013 Three dimensional flexural-gravity waves. Stud. Appl. Math. 131, 135-148.

PĂRĂU, E. I. \& DiAs, F. 2002 Nonlinear effects in the response of a floating ice plate to a moving load. J. Fluid Mech. 460, 281-305.

PĂRĂU, E. I. \& VANDEN-Broeck, J.-M. 2011 Three-dimensional waves beneath an ice sheet due to a steadily moving pressure. Trans. Phil. R. Soc. A 369, 2973-2988.

PAGE, C. \& PĂRĂU, E. I. 2014 Hydraulic falls under a floating ice plate due to submerged obstructions. J. Fluid Mech. 745, 208-222.

PeAke, N. 2001 Nonlinear stability of a fluid-loaded elastic plate with mean flow. J. Fluid Mech. 434, 101-118.

Plotnikov, P. I. \& Toland, J. F. 2011 Modelling nonlinear hydroelastic waves. Phil. Trans. R. Soc. A 369, 2942-2956.

Saffman, P. G. 1985 The superharmonic instability of finite-amplitude water waves. J. Fluid Mech. 159, 169-174.

Squire, V. A., Hosking, R. J., Kerr, A. D. \& Langhorne, P. J. 1996 Moving loads on ice plate. Solid Mechanics and its Applications, vol. 45. Dordrecht, The Netherlands: Kluwer Academic Publishers.

Squire, V. A., Robinson, W. H., Langhorne, P. J. \& Haskell, T. G. 1988 Vehicles and aircraft on floating ice. Nature 333, 159-161.

Schwarts, L. W. \& VAnden-Broeck, J.-M. 1979 Numerical solution of the exact equations for capillary-gravity waves. J. Fluid Mech. 95, 119-139.

TAKizAWA, T. 1985 Deflection of a floating sea ice sheet induced by a moving load. Cold Reg. Sci. Tech. 11, 171-180.

Toland, J. F. 2007 Heavy hydroelastic travelling waves. Proc. R. Soc. Lond. A. 463, 23712397.

Toland, J. F. 2008 Steady periodic hydroelasstic waves. Arch. Ration. Mech. Anal. 289, 325362.

VANDEN-Broeck, J.-M. \& DiAs, F. 1992 Gravity-capillary solitary waves in water of infinite depth and related free-surface flows. J. Fluid Mech. 240, 549-557.

VAnden-Broeck, J.-M. \& PĂRĂU, E. I. 2011 Two-dimensional generalized solitary and periodic waves under an ice sheet. Phil. Trans. R. Soc. A 369, 2957-2972.

Wang, Z., Vanden-Broeck, J.-M. \& Milewski, P. A. 2013 Two-dimensional flexural-gravity waves of finite amplitude in deep water. IMA J. Appl. Math. 78, 750-761.

Wang, Z., Vanden-Broeck, J.-M. \& Milewski, P. A. 2014 Asymmetric gravity-capillary solitary waves on deep water. J. Fluid Mech. 759, R2.

WAng, Z. H. 2000 Hydroelastic analysis of high-speed ships. PhD Thesis, Technical University of Denmark.

Wilson, J. T. 1958 Moving loads on floating ice sheets. UMRI Project 2432, Ann Arbor, Michigan, University of Michigan Research Institute.

ZAKHAROV, V. E. 1968 Stability of periodic waves of finite amplitude on the surface of a deep fluid. J. Appl. Mech. Tech. Phys. 2, 190-194. 